\title{
EVALUATION OF ANTIMICROBIAL EFFICACY OF GREEN TEA EXTRACT IRRIGANT VERSUS PHYSIOLOGICAL SALINE FOLLOWING ROOT CANAL TREATMENT OF INFECTED PRIMARY MOLARS IN A GROUP OF EGYPTIAN CHILDREN : A RANDOMIZED CLINICAL TRIAL
}

\author{
Mai Ramadan El Sayed Salem*, Sherine Ezz El Din Taha** and Randa Youssef Abd El Gwad*
}

\begin{abstract}
Introduction Preservation of primary tooth with non-vital pulp is a major problem in pediatric dentistry. In such cases, the preferred treatment modality is pulpectomy. The objective of pulpectomy is complete removal of necrotic and irreversibly infected pulp of an affected tooth but due to limited ability of mechanical preparation to eliminate entrapped micro organisms in the canal space owing to the tortuous and complex nature of the root canal system. Thus , the use of antimicrobial agents has been recommended as an adjunct to mechanical instrumentation in deciduous teeth.

Aim The aim of the study was to assess the antimicrobial efficacy and clinical success of green tea extract irrigant versus physiological saline following root canal irrigation of infected primary molars.

Materials and Methods In the present study, antimicrobial efficacy and clinical success of green tea extract irrigant and physiological saline as control were assessed and compared with each other. The antimicrobial efficacy was evaluated against aerobic and anaerobic. The statistical analysis was done with IBM, SPSS*. Statistics version 23 for Windows. Data were explored for normality using Kolmogorov-Smirnov and Shapiro-Wilk tests. $\log ^{10}(\mathrm{CFU} / \mathrm{ml})$ showed parametric distribution, so Dependent t-test was used to compare between before and after application. Independent t-test was used to compare between different groups.

Results: Percentage of change in bacterial count of aerobic and anaerobic bacteria revealed significant difference in preference to green tea extract group. Clinical success was higher in green tea extract group.

Conclusion: Green tea extract irrigant has antimicrobial effect against aerobic and anaerobic bacteria present in the root canals of necrotic primary molars which is higher than saline. Also, green tea extract irrigation showed higher clinical success rate than saline.
\end{abstract}

Keywords: Green tea, Saline, Antimicrobial effect, Primary teeth, Pulpectomy, Irrigation

\footnotetext{
* Master Student in Pediatric Dentistry and Dental Public Health Department, Faculty of Oral and Dental Medicine, Cairo University. ** Professor in Pediatric Dentistry and Dental Public Health Department, Faculty of Oral and Dental Medicine, Cairo University. *** Lecturer in Pediatric Dentistry and Dental Public Health Department, Faculty of Oral and Dental Medicine, Cairo University.
} 


\section{INTRODUCTION}

Tooth decay is a common phenomena among children as a result of bad eating habits and deficient oral hygiene. Treatment of primary teeth with caries lesions is essential to maintain the teeth in the arch and to reestablish the healthy condition of the tissues affected by the caries and preserving the normal development of the permanent successor teeth ${ }^{(1)}$.

Because primary teeth have thinner enamel and dentin and broader proximal contacts than permanent teeth leading to increased caries susceptibility and more rapid progression of caries to the pulp which could cause painless dento-alveolar infection leading to serious dental and systemic consequences. And failure to treat carious primary teeth markedly increased the risk of sepsis which making intervention to treat carious primary teeth is mandatory to avoid serious complications ${ }^{(2,3)}$.

Accepted endodontic therapy for primary teeth divided into vital pulp therapy and root canal treatment . Vital pulp therapy in deciduous teeth aim to maintain pulp vitality and function . While the aim of root canal treatment is to eliminate bacteria and prevent their further entry to the root canal system $^{(4)}$.

The problem is that root canal morphology of primary teeth especially posterior primary teeth contain many ramifications and deltas between canals making mechanical debridement alone is not enough for achieving this target. The use of antimicrobial agents have to be used as adjunct to mechanical instrumentation to reduce the numbers of micro-organisms in inaccessible areas ${ }^{(5)}$.

Many antiseptic and antibacterial irrigating solutions as Sodium hypochlorite $(\mathrm{NaOCl})$ ,Chlorhexidine (CHX), Ethylenediamine tetraacetic acid (EDTA) were used beside mechanical debridement to facilitate removal of necrotic pulpal tissues and disinfection of root canals ${ }^{(6)}$.
Due to potential side effects, safety concerns of synthetic drugs and cytotoxic reactions of commercial intracanal irrigants and their limited ability to eliminate bacteria from dentinal tubules. Herbal products can be an alternative to commercially available chemical formulated irrigants based on their previous history in medical field ${ }^{(7)}$.

As green tea polyphenols have significant anti-oxidant, anti-cariogenic, anti-inflammatory, thermogenic, probiotic and anti-microbial properties, along with ease of availability, economical, longer shelf-life, little or minimal toxicity and lack of microbial resistance, for thus green tea polyphenols are preferred over the traditional root canals irrigants which makes it an excellent alternative to the currently available endodontic irrigants and can be used for better disinfection of root canal system, predictable long term success of the root canal treatment and prevention of reinfection ${ }^{(8)}$.

Thus, the aim of the study was to evaluate antimicrobial effect of green tea extract as root canal irrigant of necrotic primary teeth in comparison to saline against aerobic and anaerobic bacteria and clinical success rate.

\section{MATERIALS AND METHODS}

This study was carried out in the Pediatric Dentistry and Dental Public Health Department, Faculty of Oral and Dental Medicine, Cairo University. Subjects were patients requiring root canal treatment for infected primary molars. Approval of the study from the Research Ethical Committee in Faculty of Oral and Dental Medicine, Cairo University and Pediatric Department Evidence Based Committee, Faculty of Oral and Dental Medicine, Cairo University were obtained.

A total sample size consist of 16 primary molars ( 8 in each group) was sufficient to detect large effect size ( $\mathrm{f}=1.3$ ) with power $96 \%$ and significance level 5\% regarding evaluation of the antimicrobial effect of green tea extract irrigant and saline 
(control) on infected primary molars Based on "Comparative evaluation of antimicrobial activity of miswak, propolis, sodium hypochlorite and saline as root canal irrigants by microbial culturing and quantification in chronically exposed primary teeth" ${ }^{(9)}$. Done by $\mathrm{G}^{*}$ power Version $(3.1 .9 .2)$ for windows.

To increase the power of the study and overcome dropouts if occurred study sample included 26 non-vital mandibular primary molars (D or E) with infected pulp indicated for pulpectomy . Group A $(n=13)$ received $3.5 \%$ extract of green tea as root canal irrigant ( test group ). Group B ( $n=13)$ received $0.9 \%$ saline as root canal irrigant (negative control group) .

\section{Inclusion criteria :}

Patient included in this study were selected according to following criteria :

1- The children age range from 4 years to 7 years from both sexes.

2- Presence of localized swelling, sinus and pain on percussion which indicate non vital pulp .

3- Presence of periapical and furcation radiolucency in periapical radiograph.

4- Absence of advanced physiologic or pathologic root resorption in X-ray.

5- Absence of history of systemic disease which would contraindicate pulp therapy e.g. infective endocarditis.

6- No previous history of antibiotics administration or last dose was taken from at least 2 weeks.

\section{Exclusion criteria :}

1- Presence of advanced physiologic or pathologic root resorption in X-ray.

2- Uncooperative children.

3- Teeth with unrestorable crowns.

4- Teeth with abnormal anatomy and calcified canals.
At the patient examination appointment, the nature of the study, the treatment plan, the procedure, possible discomfort or risk as well as benefits were fully explained to the parents of the children involved in the study. Parents who were unable or unwilling to participate in this study were excluded. Patients who were chosen to be included in the study, their parents were asked to sign informed consent

\section{Diagnosis :}

1- History taking from the child and parent including detailed personal, medical and dental history.

2- Clinical examination using mirror and probe .

3- Radiographic examination using preoperative periapical X-ray to assess the presence of periapical or furcation radiolucency.

4- Intra-oral preoperative photographs were taken.

\section{Preparation of the green tea extract dilutions}

Green tea extract were obtained in the form of powder which is readily soluble in water and prepared in the concentration of $3.5 \%$. Mixing $35 \mathrm{~g}$ of green tea extract with $100 \mathrm{ml}$ of sterile boiling distilled water for 5 minutes were done and then filtered by filter paper. The dilutions were kept overnight at $37^{\circ} \mathrm{C}^{(10)}$.

\section{Clinical procedure:}

A standardized protocol was followed:

- Pre-operative radiograph was taken by size 2 $\mathrm{x}$-ray film, showing all roots and their apices using Planmeca x-ray machine.

- Inferior alveolar nerve block was administrated to the patient using Septocaine with epinephrine 1:100,000.

- Involved teeth were isolated with rubber dam sheet to minimize bacterial contamination of the treatment site. 
- Caries was removed and the access to pulp chamber was gained using \#330 high speed round shaped carbide bur with coolant. Then roof of the pulp was removed with tapered diamond stone.

- The working lengths of root canals were estimated by apex locator.

- Cleaning and shaping of the root canals was done by $\mathrm{K}$ files size 20\#, 25\#, 30\# respectively (not greater than size 30\#) .

\section{Sampling procedure}

\section{First microbial sample}

The pre-irrigation sample (sample number 1) were collected after debridement of the radicular pulp tissues by a sterile paper point from distal canal.

- Paper point was placed in the canal to the apical foramen and moved circumferentially along the walls of the canal for 1 minute.

- Sterile tweezer was used to remove the paper point from the root canal and immediately placed into sterile air tight marked vials containing Thioglycolate broth for microbiological evaluation.

\section{Second microbial sample}

- The canals were irrigated with one of irrigating solutions, either $3.5 \%$ green tea extract (group A) or $0.9 \%$ saline (group B).

- Then the canals were dried with sterile paper points.

- Second microbial sample of the root canal contents after irrigation were obtained using another sterile paper point for microbiological evaluation as before .

- Root canals were obturated down to the root apex by injectable $\mathrm{Ca}(\mathrm{OH})_{2}$ with iodoform paste (Metapex) and radiograph was obtained to determine the quality of obturation.

- Tooth is then packed with IRM.

- Patient were recalled after 1 week for assessment of post-operative pain then tooth preparation and stainless steel crown were cemented if there is no complains.

\section{Microbiological culturing procedure}

All the microbiological culturing procedures were carried out in the Microbiology and Immunology Department, Faculty of Medicine, Cairo University. Agar plates were prepared by using brain-heart infusion (BHI) agar according to the manufacturer's directions. Each vial was shaken using rotary shaker to disperse the sample contents evenly. Under strict conditions of asepsis, the homogenously dispersed material in the vials was streaked on to two Petri plates containing brain heart agar. One Petri plate was incubated in electric incubator, aerobically for 48 hours at $37^{\circ} \mathrm{C}$ and one Petri plate was incubated anaerobically for 48 hours at $37^{\circ} \mathrm{C}$. Anaerobic gas generating kit were used to consume the oxygen and produce carbon dioxide in an anaerobic jar to create the anaerobic environment. After the incubation period, the plates were examined and the colonies were directly counted using colony forming unit .

\section{Post treatment evaluation :}

Assessment of outcomes clinically at intervals 1,2, 3 months according to following table: 
TABLE (1) Primary and secondary outcomes of the study, method of examination of outcomes and unitof measurement

\begin{tabular}{|l|l|l|l|}
\hline & Outcome & Tool/device & Unit \\
\hline \multirow{2}{*}{ Primary outcome } & Postoperative pain & Pain questionnaire & Binary \\
\hline \multirow{3}{*}{ Secondary outcome } & Pain on percussion & Tapping with the end of the mirror & Binary \\
\cline { 2 - 4 } & Swelling or sinus & Visual examination and palpation & Binary \\
\cline { 2 - 4 } & Tooth mobility & Applying pressure with the ends of 2 mirrors & Binary \\
\hline
\end{tabular}

TABLE (2) Materials used in the study

\begin{tabular}{|c|c|c|}
\hline Materials & Material type & Manufacturer \\
\hline Kodak & $\mathrm{X}$ - ray film & USA \\
\hline Septocaine & Local anesthesia & Septodont, USA \\
\hline Roeko flexi dam-non Latex & Rubber dam sheets & Coltene/ Whaledent, Germany \\
\hline Densply & Hand instruments, burs and stones & Tulsa Dental Specialties; USA \\
\hline Thioglycolate broth & Transporting media & LAB M, Lancashire, United Kingdom \\
\hline Brain heart infusion agar & Culture media & LAB M, United Kingdom \\
\hline RomiApex A-15 & Apex locator & UK \\
\hline IRM & Intermediate restorative material & DENSPLY \\
\hline Metapex & Intra-canal obturating matrial & META BIOMED; Korea \\
\hline Glass ionomer cement & Cementation material & Zhejiang, China (Mainland) \\
\hline $3 \mathrm{M}$ crowns & Stainless steel crown & $3 \mathrm{M}, \mathrm{USA}$ \\
\hline Assistant Reamix & rotary shaker & China \\
\hline Precision & Electric incubator & Germany \\
\hline BD, BBL GAS PAK & Anaerobic gas generating kit & USA \\
\hline BBL GasPakGasPack Jars & anaerobic jar & USA \\
\hline
\end{tabular}




\section{RESULTS}

\section{Results of Clinical Findings Evaluation}

Two cases out of 26 were dropped outs during the follow up period, one from group (A) and one from group (B), and 24 cases succeeded to attend. The clinical outcomes of the study group and control group are illustrated in Table(3), Figure(1a-d and 2a-d).

\section{Results of Antimicrobial Activity}

The antimicrobial effect of the study and control group against aerobic and anaerobic bacteria and the percentage of change in bacterial count before and after application of study and control group regarding aerobic and anaerobic bacteria illustrated in Table (4 and 5), Figure ( 3a-d).

TABLE (3) Frequency (N) and percentage (\%) of clinical outcomes in the two groups.

\begin{tabular}{|c|c|c|c|c|c|c|c|}
\hline & & & \multicolumn{4}{|c|}{ Group } & \multirow{3}{*}{ p-value } \\
\hline & & & \multicolumn{2}{|c|}{ Group A (Green Tea Extract) } & \multicolumn{2}{|c|}{ Group B (Saline) } & \\
\hline & & & $\mathbf{N}$ & $\%$ & $\mathbf{N}$ & $\%$ & \\
\hline \multirow{2}{*}{\multicolumn{2}{|c|}{ Post-Operative Pain }} & No & 9 & $75.0 \%$ & 4 & $33.3 \%$ & \multirow{2}{*}{$0.041 *$} \\
\hline & & Yes & 3 & $25.0 \%$ & 8 & $66.7 \%$ & \\
\hline \multirow{6}{*}{ Swelling or sinus } & \multirow{2}{*}{1 Month } & No & 12 & $100.0 \%$ & 9 & $75.0 \%$ & \multirow{2}{*}{$0.217 \mathrm{NS}$} \\
\hline & & Yes & 0 & $0.0 \%$ & 3 & $25.0 \%$ & \\
\hline & \multirow{2}{*}{2 Months } & No & 12 & $100.0 \%$ & 9 & $75.0 \%$ & \multirow{2}{*}{$0.217 \mathrm{NS}$} \\
\hline & & Yes & 0 & $0.0 \%$ & 3 & $25.0 \%$ & \\
\hline & \multirow{2}{*}{3 Months } & No & 12 & $100.0 \%$ & 8 & $66.6 \%$ & \multirow{2}{*}{$0.093 \mathrm{NS}$} \\
\hline & & Yes & 0 & $0.0 \%$ & 4 & $33.3 \%$ & \\
\hline \multirow{6}{*}{ Pain on percussion } & \multirow{2}{*}{1 Month } & No & 10 & $83.3 \%$ & 2 & $16.7 \%$ & \multirow{2}{*}{$0.004 *$} \\
\hline & & Yes & 2 & $16.7 \%$ & 10 & $83.3 \%$ & \\
\hline & \multirow{2}{*}{2 Months } & No & 12 & $100.0 \%$ & 7 & $58.3 \%$ & \multirow{2}{*}{$0.04 *$} \\
\hline & & Yes & 0 & $0.0 \%$ & 5 & $41.7 \%$ & \\
\hline & \multirow{2}{*}{3 Months } & No & 12 & $100.0 \%$ & 7 & $58.3 \%$ & \multirow{2}{*}{$0.04 *$} \\
\hline & & Yes & 0 & $0.0 \%$ & 5 & $41.7 \%$ & \\
\hline \multirow{6}{*}{ Mobility } & \multirow{2}{*}{1 Month } & No & 12 & $100.0 \%$ & 11 & $91.7 \%$ & \multirow{2}{*}{$1.00 \mathrm{NS}$} \\
\hline & & Yes & 0 & $0.0 \%$ & 1 & $8.3 \%$ & \\
\hline & \multirow{2}{*}{2 Months } & No & 12 & $100.0 \%$ & 10 & $83.3 \%$ & \multirow{2}{*}{$0.460 \mathrm{NS}$} \\
\hline & & Yes & 0 & $0.0 \%$ & 2 & $16.7 \%$ & \\
\hline & \multirow{2}{*}{3 Months } & No & 12 & $100.0 \%$ & 9 & $75.0 \%$ & \multirow{2}{*}{$0.217 \mathrm{NS}$} \\
\hline & & Yes & 0 & $0.0 \%$ & 3 & $25.0 \%$ & \\
\hline
\end{tabular}


TABLE (4) Mean and standard deviation (SD) of bacterial count Log 10 (CFU/ml) before and after application of green tea extract irrigation and saline for aerobic and anaerobic bacteria

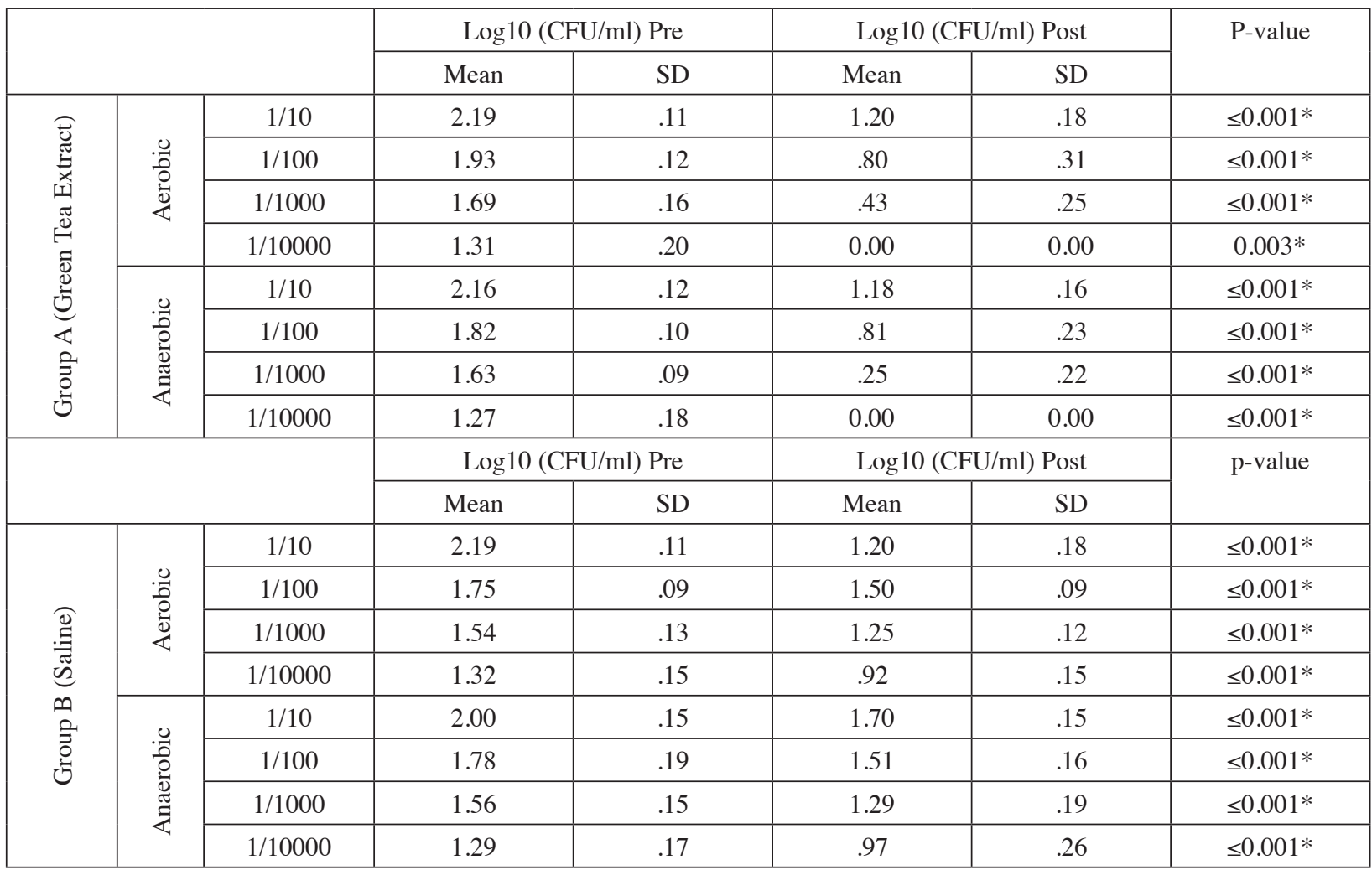

TABLE (5) Median, minimum and maximum \% of change in bacterial count Log 10 between before and after application of green tea and saline for aerobic bacteria and anaerobic bacteria.

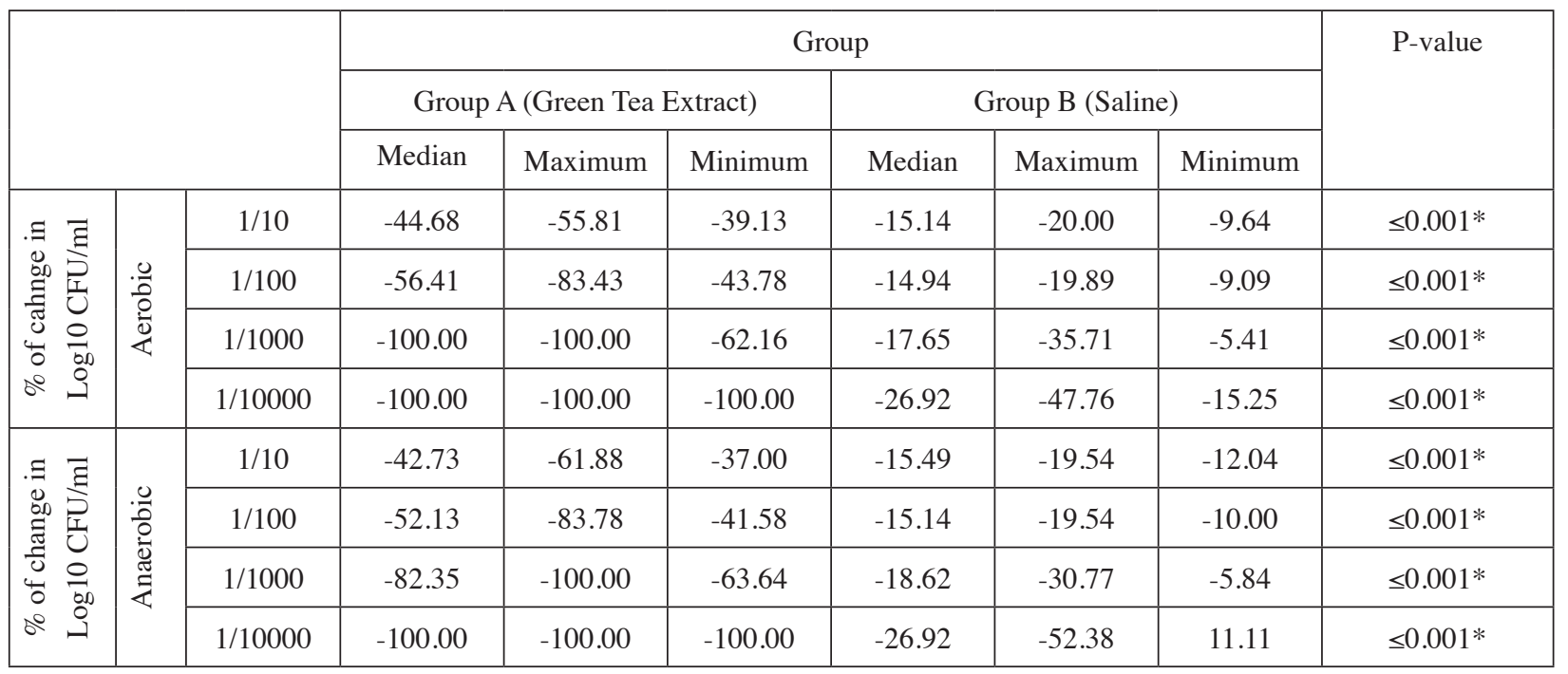

\footnotetext{
*= Significant, NS=Non-significant
} 



\section{DISCUSSION}

The endodontic therapy of primary teeth aims to maintain form and function of primary teeth ,however, complex morphology and irregularity of root canal system of primary teeth makes the use of irrigating solutions as adjunct to mechanical instrumentation is essential ${ }^{(1,5)}$.
Fig. (1) a-d: a-Preoperative photograph showing decayed lower left D and $\mathrm{E}$, and localized swelling related to lower left E . b- Postoperative photograph showing stainless steel crown restorations of lower left D and $\mathrm{E}$, and disappearance of the swelling after pulpectomy and irrigation with green tea extract (group A) after 3 months. c- Preoperative radiograph showing decayed lower left $\mathrm{D}$ and widening of lamina dura in furcation area of lower left E. d- Post operative radiograph showing root canal filling of lower left $\mathrm{E}$ after pulpectomy and irrigation with green tea extract (group A) and restored by stainless crown as final restoration.

Fig. (2) a-d: a- Preoperative photograph showing caries and localized swelling related to lower right E. bPostoperative photograph showing stainless steel crown restoration of lower right $\mathrm{E}$, and relapse of the swelling after pulpectomy and irrigation with saline ( group B) after 3 months. c- Preoperative radiograph showing decayed lower right $\mathrm{E}$ and widening of lamina dura in furcation area. d- Post operative radiograph showing root canal filling of lower right $\mathrm{E}$ after pulpectomy and irrigation with saline (group B) and restored by stainless steel crown as final restoration.

Fig. (3) a-d a-Petri dish showing bacterial colonies growing on BHI agar of first microbial sample before irrigation with green tea extract (group A). b-Petri dish showing bacterial colonies growing on BHI agar of second microbial sample after irrigation with green tea extract (group A). c-Petri dish showing bacterial colonies growing on BHI agar of first microbial sample before irrigation with saline ( group B).d- Petri dish showing bacterial colonies growing on BHI agar of second microbial sample after irrigation with saline (group B).

As natural products are more safe than chemical formulated ones, green tea extract irrigant were used for irrigation during the procedure of pulpectomy of necrotic primary molars, to evaluate the antibacterial effect and clinical success of green tea. Physiological saline was used in this study as a control as physiological saline is safe to periapical area and successor if accidentally pass beyond apical foramen ${ }^{(9)}$. 
To increase the power of the study and overcome dropouts if occurred, total of 26 non-vital mandibular primary molars ( D or E ) with infected pulp indicated for pulpectomy were included in the study. Samples were taken from distal canal of mandibular primary molars for better accessibility.

Age selection in the current study ranged from 4 years to 7 years, as this was the most favorable age group with considerable root length and cooperative patient behavior. Parent assure that no history of antibiotic coverage for at least 2 weeks for accurate and realistic antibacterial evaluation. Physiological resorption if present was confirmed to be restricted up to only one-third of the root lengths and evidence of internal or external root resorption were ruled out from the study to avoid extrusion of irrigant into periapical tissues which affect clinical signs and symptoms ${ }^{(5)}$.

Rubber dam was placed to avoid bacterial contamination of the treatment site during all procedures and filing of the root canals done by K-files not greater than size 30 to avoid over enlargement of the canals and occurrence of lateral perforation $^{(5,9,11)}$.

Clinical assessment were performed after 1,2 and 3 months postoperatively, in the present study there were two cases out of 26 were dropped out during the follow up period, one from group (A) and one from group (B).

In the present study, the clinical success of pulpectomy using $3.5 \%$ green tea extract for irrigation was higher than clinical success reported with $0.9 \%$ saline irrigation. Four cases in group B (control group) were developed swelling or sinus, also three cases in group B (control group) were developed class 2 mobility which was considered failure.

Results regarding spontaneous post operative pain and pain on percussion showed significant difference between $3.5 \%$ green tea extract irrigation (group A) and saline irrigation (group B), where green tea extract group showed less complains. This maybe due to aromatic components of green tea which showed anti-inflammatory properties in site of inflammation ${ }^{(12)}$.

The results were also consistent with Qazi and Manzoor, 2005 who showed that normal saline can be used safely for root canal irrigation and no pain occurred if absorbed accidentally in periapical area, but saline does not have anti-inflammatory properties ${ }^{(13)}$.

Results regarding swelling and mobility were not significantly differ between green tea extract irrigant (group A) and saline irrigant (group B), however group A showed less complications .

Microbial results showed that, in the $3.5 \%$ green tea extract irrigant group, there was statistically significant reduction in aerobic and anaerobic bacterial counts after irrigation, the results were in agreement with Martina et al.,2013the tube dilution method was used to determine the minimum inhibitory concentration (MIC; who showed that green tea extract had antibacterial activity against $E$. faecalis $^{(10)}$.

Results also showed that, in group B (control group), there was statistically significant reduction in aerobic and anaerobic bacterial counts after irrigation with $0.9 \%$ saline. The results of the study were consistent with the findings of Shingare and Chaugule, 2011 who observed a significant reduction in the count of bacteria in control group. This could be due to its ability to flush out debris from the root canal rather than having any antimicrobial property (9). However, this is contradict the results of Ohara et al., 1993 who found that saline was completely ineffective as an antimicrobial agent ${ }^{(14)}$.

Results regarding the \% of change in bacterial count in the two groups showed that $3.5 \%$ green tea extract irrigant has more significant antibacterial properties than $0.9 \%$ saline solution. This result agree with Madhu Pujar et al., 2011 who found 
that Triphala and green tea showed significantly better antibacterial activity against 2 -weeks biofilm than saline, and concluded that the use of herbal alternatives as a root canal irrigant might prove to be advantageous considering the several undesirable characteristics of chemical irrigants ${ }^{(15)}$.

\section{CONCLUSION}

From the results of this study, we can conclude that, green tea extract irrigant has strong antibacterial effect against aerobic and anaerobic bacteria present in the root canals of necrotic primary molars. Also saline was effective in reducing aerobic and anaerobic bacteria but significantly less than green tea extract irrigant.

Green tea extract irrigation in infected root canals of primary molars showed higher clinical success rate than saline.Post operative pain and pain on percussion following pulpectomy of primary molars significantly decreased when canals were irrigated with green tea extract irrigant in comparison to saline. Swelling and mobility after irrigation of infected root canals of primary molars with green tea extract showed better results than saline irrigation.

\section{REFERENCES}

1- Zehnder M. (2006). Root canal irrigants. Journal of Endodontics, 32(5): 389-398

2- Levine R. S., Pitts N. B. and Nugent Z. J. (2002). The fate of 1,587 unrestored carious deciduous teeth: a retrospective general dental practice based study from northern England. British Dental Journal, 193(2): 99-103.

3- Pine C. M., Harris R. V., Burnside G. and Merrett M. C. (2006). An investigation of the relationship between untreated decayed teeth and dental sepsis in 5-year-old children. British Dental Journal, 200(1): 45-47.

4- Pinkham J. R., Casamassimo P. S., Fields H. W. and McTigue DJ N. A. (2013). Pediatric Dentistry: Infancy Through Adolescence. 5th edition: 333-351.

5- Verma M. K., Pandey R. K., Khanna R. and Agarwal J. (2014). The antimicrobial effectiveness of $25 \%$ propolis extract in root canal irrigation of primary teeth. Journal of the Indian Society of Pedodontics and Preventive Dentistry, 32(2): 120-124.

6- Fedorowicz Z., Nasser M., Sequeira-Byron P., de Souza R. F., Carter B. and Heft M. (2012). Irrigants for nonsurgical root canal treatment in mature permanent teeth. The Cochrane Database of Systematic Reviews, (9), https://www.researchgate.net/publication/230842956.

7- Palombo E. A. (2011). Traditional edicinal plant extracts and natural products with activity against oral bacteria: Potential Application in the Prevention and Treatment of Oral Diseases: 1-5.

8- Pulok K., Sujay R., Sauvik B., Pratip K., Tuhin K., Utpalendu J., Srikanta P., Bishnu P. and Pradip K. (2006). Clinical study of "Triphala"- A Well Known Phytomedicine. Iranian Journal of Pharmacology and Therapeutics, 5(1):51-54.

9- Shingare P. and Chaugule V. (2011). Comparative evaluation of antimicrobial activity of miswak, propolis, sodium hypochlorite and saline as root canal irrigants by microbial culturing and quantification in chronically exposed primary teeth. Germs, 1(1): 12-21.

10- Martina L., Mohan A., Narayanan A., Sundaram M. Ebenezar A. R. and Ghani M. (2013). An in vitro comparative antibacterial study of different concentrations of green tea extracts and 2\% chlorhexidine on Enterococcus faecalis. Saudi Endodontic Journal, 3(3): 120.

11- Rodd H. D., Waterhouse P. J., Fuks A.B., Fayle S. A and Moffat M. A. (2006). Pulp therapy for primary molars. International Journal of Pediatric Dentistry, 16 (1): 15-23.

12- Cabrera C., Artacho R. and Giménez R. (2006). Beneficial effects of green tea--a review. Journal of the American College of Nutrition, 25(2): 79-99.

13- Qazi S.S. and Manzoor M.A. (2005). Comparison of post operative pain - normal saline vs sodium hypochlorite as irrigants, Pakistan Oral and Dental Journal, 25 (2):177182.

14- Ohara P., Torabinejad M. and Kettering J. D. (1993). Antibacterial effects of various endodontic irrigants on selected anaerobic bacteria. Endodontics \& Dental Traumatology, 9(3): 95-100.

15- Madhu P., Chetan P. and Ajay K. (2011). Comparison of antimicrobial efficacy of Triphala, (GTP) Green tea polyphenols and $3 \%$ of sodium hypochlorite on Enterococcus faecalis biofilms formed on tooth substrate: in vitro. Journal of International Oral Health, 3(2): 23-30. 